\title{
Persistence under Weak Disorder of AC Spectra of Quasi-Periodic Schrödinger operators on Trees Graphs
}

\author{
Michael Aizenman and Simone Warzel \\ Departments of Mathematics and Physics, Princeton University, Princeton NJ 08544, USA. \\ E-mail: aizenman@princeton.edu, swarzel@princeton.edu \\ Submitted (Mosc. Math. J.): 14 April 2005; Revised: 14 Sept 2005. \\ Dedicated to Ya. Sinai on the occasion of his seventieth birthday
}

\begin{abstract}
We consider radial tree extensions of one-dimensional quasi-periodic Schrödinger operators and establish the stability of their absolutely continuous spectra under weak but extensive perturbations by a random potential. The sufficiency criterion for that is the existence of Bloch-Floquet states for the one dimensional operator corresponding to the radial problem.
\end{abstract}

Key words. Random operators, absolutely continuous spectrum, quasi-periodic cocycles, Bloch states (2000 Mathematics Subject Classifiction: 47B80, 37E10)

\section{Contents}

1. Introduction . . . . . . . . . . . . . . . . . . 1

2. Quasi-periodic Schrödinger operators on rooted tree graphs . . . . . . . . 3

3. Bloch-Floquet eigenfunctions and the main result . . . . . . . . 5

4. Uniqueness of the covariant solution of the projective Schrödinger equation 6

5. Proof of the main result . . . . . . . . . . . . . . . 7

\section{Introduction}

In this work we consider the effects of weak disorder on the absolutely continuous (AC) spectra of tree extensions of one-dimensional Schrödinger operators $H(\theta)$ with quasiperiodic $(\mathrm{QP})$ potential. We show that contrary to the corresponding one-dimensional problem, under certain conditions AC spectra on trees persist under perturbations by weak random potentials.

The operator $H(\theta)$ is of the form

$$
H(\theta) \psi_{n}:=\psi_{n+1}+\psi_{n-1}+U\left(S^{n} \theta\right) \psi_{n},
$$


where $U: \Xi \rightarrow \mathbb{R}$ is a continuous function on a multidimensional torus $\Xi \equiv[0,2 \pi)^{\nu}$ and $S$ is the shift

$$
S \theta:=(\theta+2 \pi \alpha) \bmod 2 \pi .
$$

with a frequency vector $\alpha=\left(\alpha_{1}, \ldots, \alpha_{\nu}\right)$ for which the action of $S$ is ergodic.

To $H(\theta)$ we associate a "fanned-out" radial operator $\widehat{H}(\theta)$ which acts on the Hilbert space $\ell^{2}(\mathbb{T})$ over the vertex set $\mathbb{T}$ of a rooted regular tree graph as

$$
\widehat{H}(\theta) \psi_{x}:=\sum_{y \in \mathcal{N}_{x}} \psi_{y}+\sqrt{K} U\left(S^{|x|} \theta\right) \psi_{x} .
$$

In the above expression, the sum ranges over the set $\mathcal{N}_{x}$ of neighboring vertices of $x \in \mathbb{T}$, the distance to the root is denoted by $|x|$, and $K \geq 2$ is the branching number of the regular tree, i.e., the number of forward neighbors of each vertex. Next, we add disorder in the form of a random potential, which is given by a collection of independent and identically distributed (iid) random variables $\omega:=\left\{\omega_{x}\right\}_{x \in \mathbb{T}}$ associated with the vertices of $\mathbb{T}$. In this fashion, one obtains the ergodic operator

$$
\widehat{H}_{\lambda}(\theta, \omega) \psi_{x}=\widehat{H}(\theta) \psi_{x}+\lambda \omega_{x} \psi_{x}
$$

where $\lambda \in \mathbb{R}$ is the strength of the perturbation.

The issue of the stability of the AC spectra of ergodic radial potentials on trees was addressed in the recent work [1]. It was shown there that AC spectra do not disappear under weak disorder if for almost every $E \in \sigma_{\mathrm{ac}}(H(\theta))$ the following Schrödinger cocycle has a unique solution $\Gamma: \Xi \rightarrow \mathbb{C}^{+}$with values in the upper half-plane $\mathbb{C}^{+}$:

$$
\mathcal{A}(E, \theta) \Gamma(\theta)=\Gamma(S \theta),
$$

where $\mathcal{A}(E, \theta)$ is the Möbius transformation

$$
\mathcal{A}(E, \theta): \gamma \mapsto U(\theta)-E-\frac{1}{\gamma}
$$

As is explained below, solutions of equation (1.5) in $\mathbb{C}^{+}$are directly associated with covariant eigenstates of $H(\theta)$. For energies $E \in \sigma_{\mathrm{ac}}(H(\theta)$ one pair of conjugate eigenstates is generally obtained through the Green function, or alternatively the WeylTitchmarsh function (a generalization of this function to trees is discussed in a related context in [2]).

Here, we show that for operators with QP potentials the above condition - uniqueness of solutions of (1.5) - is satisfied whenever $H(\theta)$ admits a pair of Bloch-Floquet eigenstates with Bloch momenta outside of the countable collection of resonant values corresponding to the spectrum of the shift operator $S$. The existence of Bloch-Floquet states for QP operators is a topic which has been strongly developed since the early works by Dinaburg and Sinai [9]. In particular, the criterion required for our results was established by KAM and duality methods for a variety of cases $[9,4,22,6,10-12,5,20]$. It was also recognized that the existence of BF states is related to the issue of reducibility of the Schrödinger cocycle $[10,19,20]$. Such reducibility plays also an essential role in our main result. 


\section{Quasi-periodic Schrödinger operators on rooted tree graphs}

2.1. Remarks on the AC spectrum. Without the disorder, the AC spectrum of the fannedout operator $\widehat{H}(\theta)$ coincides with that of $H(\theta)$, and both can be characterized in term of the latter's Lyapunov exponent $\gamma(E)$ (see [3] for its definition):

Proposition 2.1. For any $Q P$ potential and any $\theta$

$$
\sigma_{\mathrm{ac}}(\widehat{H}(\theta))=\sqrt{K} \sigma_{\mathrm{ac}}(H(\theta))=\sqrt{K} \Sigma_{\mathrm{ac}},
$$

where $\Sigma_{\mathrm{ac}}$ is the (Lebesgue) essential closure of the set $\{E \in \mathbb{R}: \gamma(E)=0\}$.

Proof. It was noted in [1, Prop. A.1] that $\sigma_{\mathrm{ac}}(\widehat{H}(\theta))=\sqrt{K} \sigma_{\mathrm{ac}}\left(H^{+}(\theta)\right)$, where $H^{+}(\theta)$ is the restriction of (1.1) to the half line. For the latter, Kotani theory and the statement of independence from $\theta$ proven in [16, Thm. 6.1], ensure that

$$
\sigma_{\mathrm{ac}}\left(H^{+}(\theta)\right)=\sigma_{\mathrm{ac}}(H(\theta))=\Sigma_{\mathrm{ac}}
$$

for all $\theta$.

Remarks 2.1. (i) By the Kotani principle, the presence of an AC component in the spectrum of a one-dimensional Schrödinger operator requires its potential to be deterministic $[14,21]$. For QP potentials this principle does not stand in the way of $H(\theta)$ having AC spectrum. The existence of such a component was established for various cases; an overview can be found in $[17,12,5]$.

(ii) The most studied QP operator is the almost Mathieu operator with the potential $V_{n}(\theta)=u \cos (2 \pi \alpha n+\theta)$, at an irrational frequency $\alpha \in(0,1)$. In terms of (1.1) it corresponds to $\nu=1$, i.e., $\Xi=[0,2 \pi]$, and

$$
U(\theta)=u \cos (\theta) .
$$

As was shown in [12] for almost all $\alpha$ and $\theta$ the spectrum of $H(\theta)$ is

- pure absolutely continuous if $u<2$,

- pure singular continuous if $u=2$

- pure point with exponentially localized eigenfunctions if $u>2$.

(iii) Still with one frequency, a broader class of potentials is obtained by letting $U(\theta)=u f(\theta)$ with a real analytic, $2 \pi$-periodic function $f$. For the corresponding QP Schrödinger operators it was shown [5] that for any $\alpha$ satisfying a Diophantine non-resonance condition

$$
\sigma(H(\theta))=\sigma_{\mathrm{ac}}(H(\theta))
$$

provided $u$ is small enough.

The following observation may highlight the result presented below. By the reduction of a radial spectral problem to the one dimensional case and the above mentioned Kotani principle [14,21], the AC spectra of the fanned-out operators are unstable under the addition of arbitrarily weak radial disorder:

Proposition 2.2. If $\left\{\omega_{x}\right\}_{x \in \mathbb{T}}$ are replaced by radial iid random variables, i.e. $\omega_{x}=$ $\omega_{|x|}$, for which $\mathbb{E}\left[\log \left(1+\left|\omega_{0}\right|\right]<\infty\right.$, then the almost-sure AC spectrum vanishes,

$$
\sigma_{\mathrm{ac}}\left(\widehat{H}_{\lambda}(\theta, \omega)\right)=\emptyset
$$

for any $\lambda \neq 0$.

The main result presented here is that the response is different when the disorder is not constrained to be radial. 
2.2. A criterion for the stability of the AC spectrum. The stability criterion which was derived in [1] is expressed in terms of the uniqueness of solution of an equation associated with the Schrödinger cocycle (1.5). The equation can be viewed as describing a covariant Schrödinger eigenstate, expressed in the Ricatti form. Before explaining these concepts, let us present the criterion in an explicit form.

Proposition 2.3 ([1]). Let $\widehat{H}_{\lambda}(\theta, \omega)=\widehat{H}(\theta)+\lambda \omega$, with $\widehat{H}(\theta)$ an operator of the form (1.3) and $\omega$ an iid random potential satisfying $\mathbb{E}\left[\log \left(1+\left|\omega_{0}\right|\right)\right]<\infty$. If for Lebesguealmost all $E \in \Sigma_{\mathrm{ac}}$ the cocycle equation associated with $\widehat{H}(\theta)$ :

$$
\Gamma(\theta)=\frac{1}{U(\theta)-E-\Gamma(S \theta)}
$$

has not more than one measurable solution for a function $\Gamma: \Xi \rightarrow \mathbb{C}^{+}$with $\operatorname{Im} \Gamma \geq 0$, then the almost-sure $A C$ spectrum of $\widehat{H}_{\lambda}(\theta, \omega)$ is continuous at $\lambda=0$, in the sense that for every Borel subset $I \subseteq \Sigma_{\mathrm{ac}}$

$$
\lim _{\lambda \rightarrow 0}\left|\sigma_{\mathrm{ac}}\left(\widehat{H}_{\lambda}(\theta, \omega)\right) \cap \sqrt{K} I\right|=\sqrt{K}|I|,
$$

where $|\cdot|$ denotes Lebesgue measure.

To shed some light on (2.6), which is equivalent to (1.5), let us note that the Schrödinger equation $\phi_{n-1}+\phi_{n+1}+\left(V_{n}-E\right) \phi_{n}=0$, can equivalently be expressed in terms of the Ricatti variables $\gamma_{n}:=-\phi_{n} / \phi_{n-1}$ as:

$$
\gamma_{n}=\frac{1}{V_{n}-E-\gamma_{n+1}} .
$$

If the eigenstate state $\phi$ can be chosen as a covariant function of $\theta$, then $\Gamma:=\gamma_{0}[\phi]$ will satisfy (2.6). By a covariant eigenstate we mean here a generalized eigenfunction whose $\theta$-dependence is such that

(i) $H(\theta) \phi(\theta)=E \phi(\theta)$,

(ii) $\phi_{n+1}(\theta)=e^{i \kappa(\theta)} \phi_{n}(S \theta)$ for some measurable $\kappa: \Xi \rightarrow \mathbb{R}$,

where (i) is to be taken in the weak sense, as appropriate for a generalized eigenfunction.

As an aside, let us note that the converse is also true. More precisely, if $\Gamma: \Xi \rightarrow \mathbb{C}^{+}$ is a solution of (2.6) with $\operatorname{Im} \Gamma>0$ then

$$
\phi_{-1}(\theta):=\frac{1}{\sqrt{\operatorname{Im} \Gamma(\theta)}}, \quad \phi_{0}(\theta):=\phi_{-1}(\theta) \Gamma(\theta)
$$

determine a covariant eigenstate of $H(\theta)$, as was noted already in [7].

The difference between a covariant eigenstate and a covariant eigenfunction is significant. States are interpreted as rays, for which the phase factor in (ii) has no effect. Covariant eigenfunctions, interpreted as solutions of (i) with $\kappa=0$ in (ii) may not exist, or exist only at isolated energies. However, solutions with constant $\kappa$ do occur, and form the appropriate generalization of the Bloch-Floquet states to QP operators. We present their definition in the next section, where we also state our main result. 
Remark 2.1. For the analysis of [1] it was relevant that the diagonal element of the Green function of $H^{+}(\theta)$,

$$
\Gamma(E, \theta)=\left(H^{+}(\theta)-E-i 0\right)^{-1}(0,0),
$$

is a solution of (2.6) with $\operatorname{Im} \Gamma(E, \theta)>0$ for almost every $E \in \sigma_{\mathrm{ac}}(H(\theta))$. The proof of Proposition 2.3 proceeds by establishing that in any limit $\lambda, \eta \rightarrow 0$ the distribution of the diagonal Green function $\widehat{\Gamma}_{\lambda}(E+i \eta, \theta, \omega)$ of $\widehat{H}_{\lambda}(\theta, \omega)$ is of vanishing width, in the dependence on $\omega$ [1]. The subtle point is that the values may still depend on the way $\lambda$ and $\eta$ approach their limit. Any accumulation point satisfies the co-cycle equation (2.6), but that in itself does not yet allow to conclude that it coincides with $K^{-1 / 2} \Gamma(E / \sqrt{K}, \theta)$. However, the uniqueness of solution which is discussed here guarantees the distributional convergence of $\widehat{\Gamma}_{\lambda}(E+i \eta, \theta, \omega)$, and by implication the continuity of the AC spectum.

\section{Bloch-Floquet eigenfunctions and the main result}

Definition 3.1. $A$ Bloch-Floquet (BF) eigenfunction of $H$ with energy $E \in \mathbb{R}$ and quasi-momentum $k \in(-\pi, \pi]$ is a non-vanishing function $\psi: \mathbb{Z} \times \Xi \rightarrow \mathbb{C}$ with the properties:

(i) $H(\theta) \psi(\theta)=E \psi(\theta) \quad$ (in the weak sense).

(ii) $\psi_{n}(\theta)=e^{i k n} \varphi\left(S^{n} \theta\right)$ for some continuous $\varphi: \Xi \rightarrow \mathbb{C}$.

We say that $\psi$ forms part of a conjugate $\mathrm{BF}$ pair iff $\psi(\theta)$ and $\overline{\psi(\theta)}$ are linearly independent for almost every $\theta$.

Remarks 3.1. (i) The second requirement in the above definition is equivalent to the covariance property

$$
\psi_{n+1}(\theta)=e^{i k} \psi_{n}(S \theta) .
$$

(ii) Bloch-Floquet eigenfunctions come naturally in pairs. Namely, if $\psi$ is one then its complex-conjugate $\bar{\psi}$ is also a BF eigenfunction with energy $E$ and reversed quasimomentum. Their Wronskian

$$
[\psi, \bar{\psi}](\theta):=\psi_{0}(\theta) \overline{\psi_{-1}(\theta)}-\overline{\psi_{0}(\theta)} \psi_{-1}(\theta),
$$

is independent of $\theta$. Therefore, if $\psi(\theta)$ and $\overline{\psi(\theta)}$ are linearly independent for some $\theta$, they are linearly independent for all $\theta$ and $[\psi, \bar{\psi}] \neq 0$. This implies that the ratio

$$
\gamma(\theta):=-\frac{\psi_{0}(\theta)}{\psi_{-1}(\theta)}
$$

is well-defined and takes values with either $\operatorname{Im} \gamma(\theta)>0$ or $\operatorname{Im} \gamma(\theta)>0$ for all $\theta$.

Our main result, whose proof is presented below, is that the existence of a conjugate BF pair of $H$ at almost all energies in $I$ allows one to conclude that the criterion of Proposition 2.3 is met:

Theorem 3.1. In the situation of Proposition 2.3 let $I \subseteq \Sigma_{\mathrm{ac}}$ be a Borel set such that at Lebesgue-almost all $E \in I, H$ admits a conjugate pair of Bloch-Floquet states. Then (2.7) holds. 
The condition assumed in the above statement was established for various examples of QP operators $H$, for almost all energies in their AC spectra $[9,4,22,6,10,18,11,12$, $5,20]$. In particular, it was proven to hold for the almost Mathieu operator and the family of real analytic QP potentials mentioned in Remarks 2.1(ii) and 2.1(iii).

In view of the above, it is natural to ask whether all quasi-periodic operators admit conjugate BF pairs for almost every $E \in \Sigma_{\text {ac }}$. This question was raised in [7], and was disproved in [15].

\section{Uniqueness of the covariant solution of the projective Schrödinger equation}

\subsection{Reducibility and uniqueness. The main observation leading to Theorem 3.1 is:}

Lemma 4.1. Assume that $H$ admits a conjugate BF pair with energy $E$ and quasimomenta $\pm k$. Then:

(i) The Möbius transformation $\mathcal{A}(E, \theta)$ is reducible to a constant co-cycle, in the sense that there exists a Möbius mapping $\mathcal{Z}(\theta)$ such that

$$
\mathcal{A}(E, \theta) \mathcal{Z}(\theta)=\mathcal{Z}(S \theta) e^{-2 i k} .
$$

An explicit expression for $\mathcal{Z}(\theta)$, in terms of the $B F$ state $\psi$, is

$$
\mathcal{Z}(\theta): \gamma \mapsto \gamma=\frac{\overline{\psi_{0}(\theta)} \gamma-\psi_{0}(\theta)}{-\overline{\psi_{-1}(\theta)} \gamma+\psi_{-1}(\theta)}
$$

(ii) If $|k| / \pi \notin\left\{(m \cdot \alpha) \bmod \mathbb{Z}: m \in \mathbb{Z}^{\nu}\right\}$, then (1.5) has a unique solution with values in $\mathbb{C}^{+}$.

To place this statement in its proper context let us note that the dynamical system perspective is already known to provide considerable insight on the spectral properties of QP Schrödinger operators in one (space) dimension. In the form that is seen more often, one associates to $H(\theta)$ and a given energy $E$ the QP Schrödinger cocycle $(A(E, \cdot), \alpha)$ on $S L(2, \mathbb{R}) \times \Xi$ with

$$
A(E, \theta):=\left(\begin{array}{cc}
E-U(\theta) & -1 \\
1 & 0
\end{array}\right)
$$

In particular, it was recognized that the question of reducibility of this cocycle plays an important role in the spectral theory of $1 \mathrm{D}$ operators (see $[10,19,20]$ and references therein). The following proposition is a combination of known statements contained in [20]. In essence, its second part relates the integrated density of states (IDS) $n(E)$ of $H(\theta)$ (see [3] for a definition) to the rotation number of the Schrödinger cocycle [8].

Proposition 4.1 (cf. [20]). Assume that $H$ admits a conjugate BF pair with energy $E$ and quasi-momenta $\pm k$. Then:

(i) the Schrödinger cocycle is reducible to a constant matrix,

$$
A(E, \theta) Z(\theta)=Z(S \theta)\left(\begin{array}{cc}
e^{-i k} & 0 \\
0 & e^{i k}
\end{array}\right)
$$

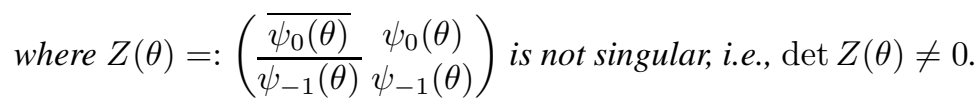


(ii) there exists some $m \in \mathbb{Z}^{\nu}$ such that

$$
\frac{|k|}{2 \pi}=\left(\frac{n(E)}{2}+m \cdot \alpha\right) \bmod \mathbb{Z},
$$

where $n(E)$ is the IDS of $H(\theta)$.

Remark 4.1. If $H$ admits a conjugate pair of covariant eigenstates, which according to (2.9) holds for almost every $E \in \sigma_{\mathrm{ac}}(H(\theta))$, then (4.4) remains true if $k$ is replaced by $\kappa(\theta)$.

The Möbius transformation $\mathcal{A}(E, \theta)$ given by (1.6) is just a projective counterpart of $A(E, \theta)$. In view of Proposition 4.1 it is therefore not surprising that $\mathcal{A}(E, \theta)$ is reducible to a constant rotation in the complex plane if $H$ has a conjugate BF pair. The main observation in Lemma 4.1 is therefore the fact that this reducibility ensures the uniqueness of solution of (1.5).

Proof (of Lemma 4.1).

(i) The reducibility (4.1) is a projective version of (4.4).

(ii) As was noted before, $\gamma(\theta):=-\psi_{0}(\theta) / \psi_{-1}(\theta)$ satisfies (1.5) and according to Remark 3.1(ii) we may assume without loss of generality that $\operatorname{Im} \gamma(\theta)>0$ for all $\theta$. Suppose now there exists yet another solution $\widetilde{\gamma} \neq \gamma$ of $(1.5)$ with values in $\mathbb{C}^{+}$. Then

$$
f(\theta):=\mathcal{Z}(\theta)^{-1} \widetilde{\gamma}(\theta)=\frac{\psi_{-1}(\theta)}{\overline{\psi_{-1}(\theta)}} \frac{\widetilde{\gamma}(\theta)-\gamma(\theta)}{\widetilde{\gamma}(\theta)-\overline{\gamma(\theta)}}
$$

satisfies

$$
(\mathcal{S} f)(\theta):=f(S \theta)=\exp [-2 i k] f(\theta)
$$

where we have introduce the (unitary) Koopmann operator $\mathcal{S}: L^{2}(\Xi) \rightarrow L^{2}(\Xi)$ associated with the ergodic shift $S$. For a QP shift with frequency $\alpha$ its spectrum consists of the countable set

$$
\operatorname{spec}(\mathcal{S})=\left\{\exp (2 \pi i m \cdot \alpha): m \in \mathbb{Z}^{\nu}\right\} .
$$

According to (4.7), $f$ is a proper eigenfunction of the Koopmann operator $\mathcal{S}$. Since $\exp [ \pm 2 i|k|] \notin \operatorname{spec}(\mathcal{S})$ this implies that $f=0$.

\section{Proof of the main result}

We are now ready to complete the prove of our main result.

Proof (of Theorem 3.1). In view of Proposition 2.3, Proposition 4.1(ii) and Lemma 4.1(ii), it remains to show that for almost all $E \in \Sigma_{\text {ac }}$

$$
n(E) \notin\left\{(m \cdot \alpha) \bmod \mathbb{Z}: m \in \mathbb{Z}^{\nu}\right\} .
$$

But this follows from the fact that $\Sigma_{\mathrm{ac}}$ is a set of positive Lebesgue measure on which the integrated density of states is not constant [3]. Since the right side in (5.1) is a countable set, it cannot coincide with the image of $\Sigma_{\text {ac }}$ under the map $E \mapsto n(E)$.

Remark 5.1. By the gap-labeling theorem $[13,8]$ the condition $n(E)=(m \cdot \alpha) \bmod \mathbb{Z}$, for some $m \in \mathbb{Z}^{\nu}$, characterizes the spectral gaps of $H(\theta)$. 
Let us end by noting that the result of [1] was derived under a weaker condition than independence for the disorder variables $\omega$. Rather, it suffices there to assume only weak correlations for the joint distributions along any two disjoint forward subtrees of $\mathbb{T}$. The results presented here are directly applicable also to this generalization.

Acknowledgement. We thank Robert Sims, Svetlana Jitomirskaya, Michael Goldstein and Uzy Smilansky for stimulating discussions of topics related to this work. MA thanks for the gracious hospitality enjoyed at the Weizmann Institute. This work was supported in parts by the Einstein Center for Theoretical Physics and the Minerva Center for Nonlinear Physics at the Weizmann Institute, by the US National Science Foundation, and by the Deutsche Forschungsgemeinschaft.

\section{References}

1. M. Aizenman, R. Sims and S. Warzel. Stability of the absolutely continuous spectrum of random Schrödinger operators on tree graphs. Preprint math-ph/0502006.

2. M. Aizenman, R. Sims and S. Warzel. Absolutely continuous spectra of quantum tree graphs with weak disorder. Preprint math-ph/0504039.

3. J. Avron and B. Simon. Almost periodic Schrödinger operators II. The integrated density of states. Duke Math. J. 50:369-391, 1983.

4. J. Bellissard, R. Lima and D. Testard. A metal-insulator transition for the almost Mathieu model. Commun. Math. Phys. 88:207-234, 1983.

5. J. Bourgain and S. Jitomirskaya. Absolutely continuous spectrum for $1 D$ quasiperiodic operators. Invent. Math. 148:453-463, 2002.

6. V. Chulaevsky and F. Delyon. Purely absolutely continuous spectrum for almost Mathieu operators. $J$. Stat. Phys. 55:1279-1284, 1989.

7. P. Deift and B. Simon. Almost periodic Schrödinger operators III. The absolutely cotinuous spectrum in one dimension. Commun. Math. Phys. 90:389-411, 1983.

8. F. Delyon and B. Souillard. The rotation number for finite difference operators and its properties. Commun. Math. Phys. 146:447-482, 1992.

9. E. Dinaburg and Y. Sinai. The one-dimensional Schrödinger equation with quasi-periodic potential. Funkt. Anal. i. Priloz. 9:8-21, 1995.

10. L. H. Eliasson. Floquet slutions for the 1-dimensional quasiperiodic Schrödinger equation. Commun. Math. Phys. 146:447-482, 1992.

11. A. Y. Gordon, S. Jitomirskaya, Y. Last and B. Simon. Duality and singular continuous spectrum in the almost Mathieu equation. Acta Math. 178:169-183, 1997.

12. S. Jitomirskaya. Metal-insulator transition for the almost Mathieu operator. Ann. Math. 150:1159-1175, 1999.

13. R. Johnson and J. Moser. The rotation number for almost periodic potentials. Commun. Math. Phys. 84:403-438, 1982.

14. S. Kotani. Ljapunov indices determine absolute continuous spectra of stationary one dimensional Schrödinger operators. In K. Ito, editor, Proc. Taneguchi Itern. Symp. on Stochastic Ananlysis, pages 225-247, Amsterdam, 1983. North Holland.

15. Y. Last. A relation between a.c. spectrum of ergodic Jacobi matrices and the spectra of periodic approximants. Commun. Math. Phys. 151:183-192, 1993.

16. Y. Last and B. Simon. Eigenfunctions, transfer matrices, and absolutely continuous spectrum of onedimensional Schrödinger operators. Invent. Math. 151:329-367, 1999.

17. L. Pastur and A. Figotin. Spectra of random and almost-periodic operators. Springer, Berlin, 1992.

18. J. Puig. Cantor spectrum for the almost Mathieu operator. Commun. Math. Phys. 244:297-309, 2004.

19. J. Puig. Reducibility of quasi-periodic skew-products and the spectrum of Schrödinger operators. $\mathrm{PhD}$ Thesis, University of Barcelona, 2004.

20. J. Puig. A non-perturbative Eliasson's reducibility theorem. Preprint math.DS/0503356.

21. B. Simon. Kotani theory for one-dimensional Jacobi matrices. Commun. Math. Phys., 89:227-234, 1983.

22. Y. G. Sinai. Anderson localization for one-dimensional difference Schrödinger operator with quasiperiodic potential. J. Stat. Phys. 46:861-909, 1987. 